\title{
Transportation Risk Control of Waste Disposal in the Healthcare System with Two-Echelon Waste Collection Network
}

\author{
Haolin Li $\mathbb{D}^{1},{ }^{1}$ Yi Hu $\mathbb{D},{ }^{2}$ Junyan Lyu $\mathbb{D},{ }^{1}$ Hao Quan $\mathbb{D}^{\circ},{ }^{2}$ Xiang Xu $\mathbb{D}^{1},{ }^{1}$ and Chenxi Li $\mathbb{D}^{1}$ \\ ${ }^{1}$ School of Management, Shanghai University, Shanghai, China \\ ${ }^{2}$ Shibei Hospital, Jing'an District, Shanghai, China \\ Correspondence should be addressed to Hao Quan; quan_hao_2001@hotmail.com
}

Received 21 January 2021; Revised 18 February 2021; Accepted 26 March 2021; Published 9 April 2021

Academic Editor: Thomas Hanne

Copyright (c) 2021 Haolin Li et al. This is an open access article distributed under the Creative Commons Attribution License, which permits unrestricted use, distribution, and reproduction in any medium, provided the original work is properly cited.

This paper investigates a vehicle routing problem arising in the waste collection of the healthcare system with the concern of transportation risk. Three types of facilities abstracted from the health system are investigated in this paper, namely, facilities with collection points, facilities without collection points, and small facilities. Two-echelon collection mode is applied in which the waste generated by small facilities is first collected by collection points, and then transferred to the recycling centre. To solve this problem, we propose a mixed-integer linear programming model considering time windows and vehicle capacity, and we use particle swarm optimisation (PSO) algorithm for solving large-scale problems. Numerical experiments show the capability of the proposed algorithm. Sensitivity analysis is conducted to investigate the influence of facilities with collection points and the collection routes. This research can provide a decision support tool for the routing of waste collection in the healthcare system.

\section{Introduction}

As COVID-19 spreads across the globe, healthcare systems are under enormous pressure. The healthcare system needs to treat more patients and prevent the spread of diseases, as well as dispose of the waste generated by the infected victims. The waste is more hazardous to the public than other common solid waste as it is infectious and toxic [1]. The waste can become a source of infection and thus spread disease if the waste is not properly disposed of. Consequently, the storage and transportation of waste generated by the healthcare system pose a potential risk to the public. All the steps need to be carefully planned, processed, and monitored so that the potential risks can be eliminated.

In a highly developed healthcare system, different facilities play different roles and provide differentiated health services to residents, including hospitals, clinics, laboratories, and temporary facilities. An effective waste collection network can provide essential support for the normal and safe operation of all facilities in the healthcare system. In a typical waste collection network, several routes are assigned to collect the waste generated by the daily operation of the healthcare system, which covers all facilities of the system. Collection vehicles follow the scheduled routes and visit medical facilities to collect waste every day. To ensure the safety of transportation and simplify system operation, the waste collection routes in the network are generally fixed. Effective route planning of waste transportation is required to enhance efficiency and eliminate potential risks. In the daily operation of the network, the amount of waste produced by different facilities may vary according to the scale and function, where many small-scale facilities usually produce less waste. The collection task of small facilities can be time-consuming, whereas the amount of waste produced by small facilities accounts for a smaller proportion of the total. Besides, some temporary facilities may place an excessive burden on the network. Building a waste collection network covering all facilities could be challenging. To deal with these complexities, a two-echelon collection mode is applied by some collection network, where the waste in some facilities is directly collected, and collection points are constructed to collect and transfer the waste produced by small facilities. Under the setting of the two-echelon 
collection mode, the collection routes will only visit collection points and facilities that require direct collection.

The recycling of general wastes has been extensively studied in previous studies. The main decision problem in these works is the routing and scheduling of recycling vehicles so that all the waste can be collected economically. Generally, this problem can be formulated as a vehicle routing problem (VRP) to determine the routes of recycling vehicles. With respect to the issue of waste collection problem in the healthcare system, VRP is still worthy to be studied. Noticing the hazard of waste produced by the healthcare system, the potential transportation risk of waste collection in the healthcare system can be of vital importance in the decision-making process. A route with a shorter travelling distance or cost but higher transportation risk is unacceptable in practice. In this paper, we pay attention to the transportation risk in the waste collection network of the healthcare system and develop a method to determine the collection routes with the lowest transportation risk. A waste collection network with a two-echelon collection mode is formulated as a VRP problem in which the waste in some facilities needs to be collected and transferred by collection points. Particle swarm optimisation (PSO) is applied for problem-solving, and some management insights are obtained by sensitivity analysis.

The rest of this paper is organised as follows. Section 2 reviews the works related to waste collection. Section 3 explains the research problems in the waste collection network. Section 4 elaborates the mathematical model of waste collection. Section 5 proposes the solution method of the model. Section 6 verifies the efficiency of the proposed algorithm and shows management insights by conducting a sensitivity analysis. Section 7 summarises the research.

\section{Literature Review}

The waste collection problem in the healthcare system can be classified as a typical VRP. There are some works of VRP in the healthcare system; for example, Veenstra et al. [2] investigate the facility location problem and VRP arises in home delivery of medication in healthcare logistics. More works devoted to the collection of other wastes [3]. Among the numerous types of VRP , VRP with time windows and capacitated VRP have been widely investigated by the previous research [4-6]. The two extensions could be of importance in waste collection, which is used as the basis of our research. Van Engeland and Beliën [7] study waste collection with capacitated vehicles in time windows, in which vehicle must visit the disposal facility to discharge the waste after each customer visit. The routing decision is made to minimise total cost, and a column generation algorithm as well as a heuristic are proposed. Hannan et al. [8] study a modified PSO algorithm in a capacitated VRP model for waste collection to determine routes. Threshold waste level and scheduling concepts are applied in the process of waste collection. Delgado-Antequera et al. [9] model waste collection as a capacitated VRP with the objective of travel cost minimisation and route balancing. A multiphase heuristic procedure is applied. Montagné et al. [10] consider an inventory routing problem over a time horizon. Daily routes of vehicles and points visited for each day are determined with the cost-effectiveness perspective. Taslimi et al. [11] introduce a periodic load-dependent capacitated VRP for medical waste collection and seek to minimise the transportation risk and occupational risk. The transportation risk is dependent on the weight of medical waste traversing on an arc. The occupational risk is related to the temporary storage of hazardous waste at the healthcare centre. A decomposition-based heuristic algorithm is proposed. Tirkolaee et al. [12] investigate a sustainable multitrip location-routing problem with time windows for medical waste management. The factors include travelling time, total violation from time windows, service priorities, total infection, and environmental risk are minimised in their model. A fuzzy chanceconstrained programming approach is applied to address uncertainty. The model is then tested by a case study in Sari city of Iran.

Some works are devoted to carbon emission reduction and other environmental protection aspects $[13,14]$. In the field of waste collection, Molina et al. [15] use eco-efficiency as a performance indicator which takes carbon dioxide emissions and pollutant emissions into account for the waste collection route decision. Farrokhi-Asl et al. [16] present a multiobjective mathematical model considering a sustainable collection network for the collection, treatment, recycling, and disposal of hazardous waste. Three objectives including operational cost and social costs are considered and optimised. Wei et al. [17] develop an artificial bee colony and variable neighbourhood descend-based hybrid approach to investigate the waste collection problem. The socalled midway disposal pattern, which refers that the waste loaded in vehicles could be dumped to the nearest disposal facility dynamically, contributes to significant carbon emission reduction. Wu et al. [18] develop a green VRP model considering different priorities of waste and aim to reduce both greenhouse gas emission costs and waste management costs. Medical waste is assigned with higher priority to minimise the potential harm and risk to the environment. The proposed model can reduce the negative effect and improve system efficiency.

Other aspects of VRP for waste collection are also studied by the previous research. Aringhieri et al. [19] address the waste collection problem as a pickup and delivery VRP, where full container load with waste is picked up and replaced by empty containers. Jammeli et al. [20] propose a model to determine the vehicle routes and the number of waste bins to be assigned to each potential location and conduct a biobjective optimisation of minimising the collection costs and the environmental impact. A heuristic algorithm is designed where bins are clustered first, and routes are then decided. Mekamcha et al. [21] investigate the waste collection problem based on a case in Algeria and model the problem as a travelling salesman problem. A tabu search algorithm and a simulated annealing (SA) algorithm are used for decision making. De Bruecker et al. [22] study an integrated problem for waste collection and shift scheduling which finds minimal cost shift schedules and collection routes. A regular shift is supposed in this research 
for its lower cost. A multiobjective linear programming model for infectious medical waste reverse logistics network is developed by Kargar et al. [23]; in which costs, associated risks, and amount of uncollected medical waste are considered. For problem-solving, they provide a revised multichoice goal programming method.

As is known to all, VRP is an NP-hard problem. Heuristic algorithms are usually used to solve VRP and obtain near-optimal solutions in a reasonable time. Global search algorithms like genetic algorithm and PSO are applicable for finding the global optimum [24, 25]. For local search heuristics, some metaheuristics are applied to accelerate convergence while avoid trapping in local optimum, such as Squeaky Wheel Optimization [26, 27], Critical-Shaking Neighbourhood Search [28, 29], and column generationbased heuristic [30].

To summarise the literature reviewed above, waste collection is an emerging research field for the healthcare system and city normal operation. The decision making of the waste transferring at the collection points is still valuable to be discovered. The main contribution of our research can be categorized into three aspects. First, we investigate the VRP of the waste collecting network for the healthcare system considering the two-echelon collection mode in which some waste is transferred at collection points. A mixed-integer linear programming model is formulated to minimise the total transportation risk. Second, the PSO algorithm is used for problem-solving, which shows strong global search ability for solving real-scale problems. Third, by conducting a sensitivity analysis, we derive managerial insights about collection points and routes, which provide a broad view of network construction and operation.

\section{Problem Background}

This research aims to minimise the transportation risk of waste collection network in the healthcare system. The network is used to collect daily waste generated by healthcare facilities. Once the waste is generated, it is stored in temporary storage areas within facilities or sent to nearby collection points depending on the type of facility. The collection task is performed daily by the collection vehicles. The collection vehicles start from the recycling centre, visit assigned facilities along the assigned routes to collect waste, and go back to the recycling centre when all waste in the assigned facilities are collected.

The facilities in the healthcare system have different functions and sizes, so the amount of waste generated by different facilities varies. For those facilities that generate less waste, the direct collection of waste by the collection vehicle is usually not economical. Here we introduce collection points in the collection network and establish a two-echelon collection network for those small facilities and temporary facilities, in which the waste generated by those facilities is collected to collection points first and then transferred to the recycling centre for disposal. A way to set up collection points is to use existing facilities. The healthcare system can use waste disposing and temporary storage areas in existing facilities as waste collection points. This provides a more realistic and economical way of operating the healthcare system. This paper only takes into account the circumstance in which the collection points are located in existing facilities.

Based on the background mentioned above, we divide the facilities in the healthcare system into three categories by function and scale, namely, facility with collection point, facility without collection point, and small facilities. Facilities with collection points usually include hospitals and health centres which are densely distributed in the city. These facilities play the role of waste producer and collection point simultaneously. The waste generated by facilities with collection points is stored at their collection points and collected by collection vehicles. Meanwhile, the waste produced by small facilities is also collected and transferred by the collection points. Thus, the collection task of facilities with collection points includes the daily waste produced by the facility and the waste sent by the assigned small facilities. Different from those facilities with collection points, there is no collection point in facilities without collection points due to limited storage space and other possible reasons, and these facilities need not collect waste from small facilities. For facilities without collection points, the waste generated by themselves is stored and awaits collection in the temporary storage area located in the facilities. The collection tasks of facilities without collection points only include the daily waste produced by themselves. The small facilities mainly include clinics, residential areas, and some temporary facilities and appear as supplements to the healthcare system. Small facilities tend to generate less waste than the two types of facilities mentioned above, and the waste generated by small facilities is not collected directly by collectors. Instead, the waste is collected and transferred by nearby collection points, that is, the waste generated by small facilities is sent to collection points once generated and waits to be collected by collectors. To do this, we should determine which collection points are selected to transfer the waste generated by small facilities. The collection points can receive the waste generated by small facilities within their service area, which usually cover the area with a service radius which is usually not too long. This implies that the transport distance between small facilities and collection points is usually short. Because the transportation risk is distance-sensitive, the risk of waste collection from small facilities to collection points is a relatively small value, and we can assume that the risk is not considered in the proposed model.

The waste collection task is generated and must be completed daily. The collection vehicles follow certain routes and visit the assigned facilities for waste collection and complete the collection tasks. To avoid traffic jams and reduce the transportation risk, the transport of waste must be done at night, and the collectors work during certain working hours. Therefore, a time window is added to the collection routes. Meanwhile, the collection vehicles all have a maximum load capacity limit, and thus, the number of tasks assigned to a route is limited. The above problem setting can be abstracted as a capacitated VRP with time windows, in which the recycling centre serves as the depot of the network, the facilities with collection points and facilities 
without collection points are the nodes where demands are located, and the route between facilities and recycling centres are the arcs. The risk of each arc can be assessed based on distance, population density, and other related factors, and the risk value of an arc is known in advance. The objective of this problem is to minimise the total transportation risk, which can be calculated by the sum of all the selected arcs in the network. The decision problem contains the vehicle collection routes as well as the time of the tasks served.

\section{Mathematical Model}

4.1. Notations. The notations used to formulate the model are introduced as follows.

Indices and sets used in this study are as follows:

$i$ : index of a facility

$I$ : set of all facilities

$I_{1}$ : set of facilities with collection points

$I_{2}$ : set of facilities without collection points

$I_{3}$ : set of small facilities

$I^{0}$ : a subset of facilities set $I, I^{0} \subseteq I, 2 \leq\left|I^{0}\right| \leq|I|$

$r$ : index of a route

$R$ : set of all routes

Parameters used in this study are as follows:

$e, e^{\prime}:$ recycling centre, the start points and end points of all collection routes

$r_{i, j}$ : transportation risk on the arc from node $i$ to $j$, $i \in I_{1} \cup I_{2} \cup\{e\}, j \in I_{1} \cup I_{2} \cup\left\{e^{\prime}\right\}$

$m_{i, j}$ : travelling time on the arc from node $i$ to $j$, $i \in I_{1} \cup I_{2} \cup\{e\}, j \in I_{1} \cup I_{2} \cup\left\{e^{\prime}\right\}$

$d_{i, j}$ : set to 1 if the clinic $i$ locates within the service radius of collection point $j$, 0 otherwise, $i \in I_{3}, j \in I_{1}$

$p_{i}$ : the time of completing a collection task in facility $i$, $i \in I$

$[a, b]$ : time window of waste collection

$q_{i}$ : the amount of medical waste produced by the facility $i, i \in I$

$c_{i}$ : maximum capacity of collection point $i, i \in I_{1}$

$v_{r}$ : maximum capacity of route $r, r \in R$

$M$ : a sufficiently large positive number

Decision variables used in the study are as follows:

$\alpha_{i, j}$ : binary variable, set to 1 if the waste of small facility $i$ is transferred to the collection point $j, 0$ otherwise, $i \in I_{3}, j \in I_{1}$

$\beta_{i, r}$ : binary variable, set to 1 if the facility $i$ is served by route $r, 0$ otherwise, $i \in I_{1} \cup I_{2}, r \in R$

$\gamma_{i, j, r}$ : binary variable, set to 1 if the vehicle $r$ serves the facility $j$ immediately after serving the facility $i, 0$ otherwise, $i \in I_{1} \cup I_{2} \cup\{e\}, j \in I_{1} \cup I_{2} \cup\left\{e^{\prime}\right\}, r \in R$

$\delta_{i, r}$ : float variable, the arrival time of facility $i$ in route $r$, $i \in I_{1} \cup I_{2}, r \in R$ $\varepsilon_{i, r}$ : float variable, the amount of medical waste in facility $i$ transported by route $r, i \in I_{1} \cup I_{2}, r \in R$

$\zeta_{i}$ : float variable, the total amount of waste to be collected in facility $i, i \in I_{1} \cup I_{2}$

\subsection{Mathematical Model Construction.}

Minimise $\sum_{r \in R} \sum_{i \in I_{1} \cup I_{2} \cup\{e\}} \sum_{j \in I_{1} \cup I_{2} \cup\left\{e^{\prime}\right\}} r_{i, j} \gamma_{i, j, r}$,

s.t. $\sum_{j \in I_{1}} \alpha_{i, j}=1, \quad \forall i \in I_{3}$,

$\alpha_{i, j} \leq d_{i, j}, \quad \forall i \in I_{3}, j \in I_{1}$,

$$
\sum_{r \in R} \beta_{i, r}=1, \quad \forall i \in I_{1} \cup I_{2},
$$

$\sum_{i \in I_{1} \cup I_{2} \cup\{e\}} \gamma_{i, j, r}=\sum_{i \in I_{1} \cup I_{2} \cup\left\{e^{\prime}\right\}} \gamma_{j, i, r}=\beta_{j, r}, \quad \forall j \in I_{1} \cup I_{2}, r \in R$

$\sum_{i \in I_{1} \cup I_{2} \cup\left\{e^{\prime}\right\}} \gamma_{e, i, r}=\sum_{i \in I_{1} \cup I_{2} \cup\{e\}} \gamma_{i, e^{\prime}, r}=1, \quad \forall r \in R$,

$\sum_{i \in I^{0}} \sum_{j^{\prime} \in I^{0}} \gamma_{i, j, r} \leq\left|I^{0}\right|-1, \quad I^{0} \subseteq I, 2 \leq\left|I^{0}\right| \leq|I|, \forall r \in R$

$\zeta_{i}=q_{i}+\sum_{j \in I_{3}} q_{j} \alpha_{j, i}, \quad \forall i \in I_{1}$,

$\zeta_{i}=q_{i}, \quad \forall i \in I_{2}$,

$\zeta_{i} \leq c_{i}, \quad \forall i \in I_{1}$,

$\varepsilon_{i, r} \leq M \beta_{i, r}, \quad \forall i \in I_{1} \cup I_{2}, r \in R$,

$\varepsilon_{i, r} \leq \zeta_{i}, \quad \forall i \in I_{1} \cup I_{2}, r \in R$,

$\varepsilon_{i, r} \geq \zeta_{i}-M\left(1-\beta_{i, r}\right), \quad \forall i \in I_{1} \cup I_{2}, r \in R$,

$\sum_{i \in I} \varepsilon_{i, r} \leq v_{r}, \quad \forall r \in R$,

$\delta_{i, r} \geq a+p_{i}+M\left(\beta_{i, r}-1\right), \quad \forall i \in I_{1} \cup I_{2}, r \in R$,

$\delta_{i, r} \leq b-M\left(\beta_{i, r}-1\right), \quad \forall i \in I_{1} \cup I_{2}, r \in R$,

$\delta_{i, r} \leq M \beta_{i, r}, \quad \forall i \in I_{1} \cup I_{2}, r \in R$,

$\delta_{j, r} \geq \delta_{i, r}+p_{j}+m_{i, j}-M\left(1-\gamma_{i, j, r}\right)$,

$\forall i \in I_{1} \cup I_{2} \cup\{e\}, j \in I_{1} \cup I_{2} \cup\left\{e^{\prime}\right\}, r \in R$, 


$$
\begin{aligned}
& \alpha_{i, j} \in\{0,1\}, \quad \forall i \in I_{3}, j \in I_{1}, \\
& \beta_{i, r} \in\{0,1\}, \quad \forall i \in I_{1} \cup I_{2}, r \in R,
\end{aligned}
$$$$
\gamma_{i, j, r} \in\{0,1\}, \quad \forall i \in I_{1} \cup I_{2} \cup\{e\}, j \in I_{1} \cup I_{2} \bigcup\left\{e^{\prime}\right\}, r \in R,
$$

$$
\delta_{i, r}, \zeta_{i}, \varepsilon_{i, r} \geq 0, \quad \forall i \in I_{1} \cup I_{2} \cup\left\{e, e^{\prime}\right\}, r \in R
$$

The objective function of this model shown in (1) is to minimise the total transportation risk. Constraints (2) and (3) state the condition of the transportation between the small facilities and the facilities with collection points, that is, the waste of a small facility must be transported to only one collection point within the service area. Constraints (4) make sure that all tasks must be assigned to a route. Constraints (5)-(7) ensure the routes' consecutiveness of all routes and prevent the routes forming loops. Constraints (8) and (9) state the total amount of waste to be collected. The total amount of waste to be collected in a facility with collection point is equal to the sum of the waste generated by itself and the assigned small facilities. The total amount of waste to be collected at a facility without collection point is equal to the amount generated by itself. Constraints (10) make sure that the total amount of waste stored in a facility cannot exceed the facility's maximum capacity. Constraints (11)-(13) denote the collection amount of facility $i$ by route $r$. If facility $i$ is assigned to be collected by route $r$, the collection amount should be equal to the total amount. If facility $i$ is not assigned to route $r$, the collecting amount must be zero. Constraints (14) imply that the total amount of waste transported in a route should not exceed vehicle maximum capacity. Constraints (15) and (16) set the working time limit on routes. Constraints (17) and (18) denote the start time of collection tasks on all the facilities. Constraints (19)-(22) define all decision variables.

\section{Solution Method}

5.1. PSO Solution Method. When considering the problemsolving methodology, commercial solvers such as CPLEX seem to be capable of solving some small-scale instances. However, the feature that VRP is an NP-hard problem makes it difficult to solve the real-scale problem with commercial solvers. Observing the excellent performance of the PSO algorithm in solving VRP and other logistics problem, we apply PSO as the methodology of the proposed problem [31-33]. PSO is widely used to solve optimisation problems as it has simple feature and quick convergence [34].

For the PSO method, we choose the binary variables $\alpha_{i, j}, \beta_{i, r}$, and $\gamma_{i, j, r}$ to formulate the particles with the notation of $\eta_{m i}^{n}, \theta_{m r}^{n}$, and $\iota_{i}$. Dimensional reduction is applied for the particles $\eta_{m i}^{n}$ and $\theta_{m r}^{n}$. For a particle $m$ in iteration $n, \eta_{m i}^{n}$ denotes the number of collection point for the waste collection of small facility $i$, and $\theta_{m r}^{n}$ denotes the facility number assigned to route $r$. For the variable $\pi_{i, j, r}$, we use the priority attributes $i_{i}$ to represent the service sequence of facility $i$ on the route $r$, where the facilities with smaller number of $\iota_{i}$ will be served first, and the facilities with larger number of $t_{i}$ will be served later.

The updating formulas of velocity and position for both groups of particles are presented as follows:

$$
\begin{aligned}
\eta V_{m j}^{n+1} & =\eta V_{m j}^{n}+c_{1} r_{1}\left(\eta \text { pBest }_{m j}^{n}-\eta_{m j}^{n}\right)+c_{2} r_{2}\left(\eta \text { gBest }_{j}^{n}-\eta_{m j}^{n}\right), \\
\eta_{m j}^{n+1} & =\eta_{m j}^{n}+\eta V_{m j}^{n+1}, \\
\theta V_{m r}^{n+1} & =\theta V_{m r}^{n}+c_{1} r_{1}\left(\theta \text { pBest }_{m r}^{n}-\theta_{m r}^{n}\right)+c_{2} r_{2}\left(\theta \text { gBest }_{r}^{n}-\theta_{m r}^{n}\right), \\
\theta_{m r}^{n+1} & =\theta_{m r}^{n}+\theta V_{m r}^{n+1}, \\
\iota V_{m i}^{n+1} & =\iota V_{m i}^{n}+c_{1} r_{1}\left(\iota \text { pest }_{m i}^{n}-\iota_{m i}^{n}\right)+c_{2} r_{2}\left(\iota \text { gBest }_{i}^{n}-\iota_{m i}^{n}\right), \\
\iota_{m i}^{n+1} & =\iota_{m i}^{n}+\iota V_{m i}^{n+1} .
\end{aligned}
$$

In these formulas, the previous velocities of particles $\eta_{m i}^{n}$, $\theta_{m r}^{n}$, and $\iota_{i}$ are represented by $\eta V_{m i}^{n}, \theta V_{m r}^{n}$, and $\iota V_{m i}^{n}$, and the current velocitiesare represented by $\eta V_{m i}^{n+1}, \theta V_{m r}^{n+1}$, and $\iota V_{m i}^{n+1}$. The best particles for dimension $m$ to iteration $n$ are denoted by $\eta \mathrm{pBest}_{m j}^{n}, \theta \mathrm{pBest}_{m r}^{n}$, and $\iota \mathrm{pBest}_{m i}^{n}$. The best positions of the whole swarm until iteration nare denoted by $\eta \mathrm{gBest}_{j}^{n}$, $\theta \mathrm{gBest}_{r}^{n}$, and $\iota \mathrm{gBest}_{i}^{n}$. For all particles, $c_{1}$ and $c_{2}$ are acceleration weights; $r_{1}$ and $r_{2}$ are two random numbers generated within the interval $[0,1]$.

Since we apply the dimensional reduction in solution representation, the continuous position of particles should be restored into the binary form of decision variables. The modification is listed as follows:

$$
\begin{aligned}
& \alpha_{i, j}= \begin{cases}1, & \text { when } n \leq \eta_{m i}^{n}<n+1, \\
0, & \text { otherwise, }\end{cases} \\
& \beta_{i, r}= \begin{cases}1, & \text { when } n \leq \theta_{m r}^{n}<n+1, \\
0, & \text { otherwise. }\end{cases}
\end{aligned}
$$

5.2. Main Framework of the PSO Procedure. The PSO procedure for the proposed problem is listed in Algorithm 1.

\section{Numerical Experiment}

To verify the efficiency of the proposed algorithm and obtain some management insights, we conduct numerical experiments on a computer with Intel Xeon E5-2680 v4 CPU @2.40 GHz and $256 \mathrm{~GB}$ RAM. The proposed model and algorithm are implemented in C\# (VS2019) concert technology with the IBM ILOG CPLEX 12.6.1.

6.1. Generation of the Test Instances. In the computational experiments, we test nine instances with different scales. The scale settings for the instance groups (ISGs) are shown in Table 1. The time window for the route is from $0 \mathrm{am}$ to $10 \mathrm{am}$. The service radius of facilities with collection points is 500 metres. 
Parameters : $\mathbf{n}, \mathbf{N}, \mathbf{c}_{1}, \mathbf{c}_{2}, \mathbf{r}_{1}, \mathbf{r}_{2} / / n$ represents the current iteration number, and $N$ represents the maximum iteration number.

(1) Define $\eta_{m j}^{n}, \beta_{m r}^{n}, l_{m w}^{n}, \pi_{m r z}$, Fitness ${ }_{m}^{n}$, PBest $t_{m}^{n}$, and GBest ${ }^{n}$

(2) For $\mathbf{m} \in \mathbf{M} / / \mathbf{M}$ is the set of particles

(3) Set $\eta_{m j}^{n}, \theta_{m r}^{n}$, and $\iota_{m w}^{n}$ to a random number $i \in I_{1}, p_{j, i}=1$

(4) Reorder $\pi_{m r z}$ based on $\chi_{m w}^{n}$

(5) If $\delta_{i, r} \in\left[a^{f}, b^{f}\right], \zeta_{i} \leq c_{i}, \sum_{i \in I} \varepsilon_{i, r} \leq v_{r}$, then calculate the fitness value; otherwise calculate the fitness value with penalty cost

(6) Update PBest ${ }_{m}^{n}$, GBest ${ }^{n}$

(7) End for

(8) While $(\mathbf{n}<\mathbf{N})$ do

(9) For $\mathbf{m} \in \mathbf{M}$

(10) Update the velocity and position of $\eta_{m j}^{n}, \theta_{m r}^{n}$, and $\iota_{m w}^{n}$

(11) Reorder $\pi_{m r z}$ based on $\imath_{m w}^{n}$

(12) If $\delta_{i, r} \in\left[a^{f}, b^{f}\right], \zeta_{i} \leq c_{i}, \sum_{i \in I}^{m} \varepsilon_{i, r} \leq v_{r}$, then calculate the fitness value; otherwise calculate the fitness value with penalty cost

(13) Update PBest $t_{m}^{n}$, GBest $^{n}$

(14) End for

(15) $\mathbf{n}=\mathbf{n}+1$

(16) End while

(17) Return GBest ${ }^{n}$

Algorithm 1: PSO algorithm procedure.

The capacity of route $v_{r}$ is set to $1500 \mathrm{~kg}$ for each route, and the capacity of collection point $c_{i}$ is set to $1000 \mathrm{~kg}$. The collection task starts at $12 \mathrm{am}$ and ends at $10 \mathrm{am}$. Other input parameters are randomly generated based on the intervals given in Table 2. For the PSO algorithm, $r_{1}$ and $r_{2}$ are randomly generated within the interval $[0,1]$. According to the test results, the acceleration weights $c_{1}$ and $c_{2}$ are both set to 0.638 .

6.2. Performance of the Proposed Solution Method. To validate the quality and efficiency of the proposed PSO algorithm, we first conduct the experiments on small-scale instances and compare the results obtained by the PSO algorithm CPLEX solver. The results are listed in Table 3. The PSO algorithm performs quite well in terms of solution quality with an average gap value of $4.39 \%$. The PSO algorithm performs more efficiently than CPLEX in terms of computation time, where the average computing time for PSO is only 34.1 seconds.

When dealing with large-scale instances in our experiments, we design a set of assignment rules to generate solutions for comparison purposes. The main procedure of the rules is listed as follows:

Step 1: the waste of the small facilities is collected by the nearest collection point without exceeding the collection point's capacity. If the waste collected by a facility has reached its capacity, then find the second nearest facility.

Step 2: the total facilities for each route are proportionally distributed based on its load capacity and time windows

Step 3: based on the "Nearest Service" principle, we assign the nearest facilities that have not been assigned to a route and calculate the total travel distance and time. When the route reaches the capacity or time limit, the assignment of the route is completed. After that, we begin the assignment of the next route.
Table 4 illustrates the comparison between the proposed solution methods and the rules. As can be seen, the PSO algorithm can obtain a good solution in solving the largescale problem instances, with an average computation time of 391.6 seconds. More importantly, it is seen that the PSO algorithm outperforms the rules for all instances from ISG4 to ISG6 in terms of solution quality. The average gap between the two solutions is $-20.12 \%$, that is, the PSO algorithm can achieve further optimisation of the solution of rules in solving large-scale problems.

6.3. Sensitivity Analysis. We conduct sensitivity analysis to discover the effect of different settings on the total transportation risk. The impact of facilities with collection points and collection routes is investigated in our research. All the experiments of sensitivity analysis are conducted based on the setting of ISG4 in which only a specific parameter (or the parameters of a specific interval for parameter generation) is changed.

6.3.1. Sensitivity Analysis about Facilities with Collection Points. The sensitivity analysis of facilities with collection points cares about service area of the collection point, the waste amount generated by the facility itself, and the number of facilities with collection points. First, we investigate the impact of the service area by changing the service radius. The results are shown in Figure 1. The total transportation risk is reduced with the expansion of the service area. This is because that a large service area of collection points can provide more choices for waste collection of small facilities, and small facilities can be assigned to collection points that belong to a collection route with lower risk. Since the distance between a small facility and its assigned collection point is short, we assumed that the risk of waste collection from small facilities to collection points is a fixed value. When the service area continues to expand, the 
TABLE 1: Scale of instance groups in experiments.

\begin{tabular}{|c|c|c|c|c|}
\hline Group ID & No. of facilities without collection points & No. of facilities with collection points & No. of small facilities & No. of routes \\
\hline ISG1 & 6 & 7 & 14 & 4 \\
\hline ISG2 & 8 & 10 & 16 & 5 \\
\hline ISG3 & 10 & 14 & 20 & 6 \\
\hline ISG4 & 18 & 22 & 30 & 7 \\
\hline ISG5 & 25 & 35 & 50 & 8 \\
\hline ISG6 & 35 & 45 & 65 & 9 \\
\hline
\end{tabular}

TABLE 2: Intervals for parameter generation of the experiment.

\begin{tabular}{lccc}
\hline Parameter & Interval & Parameter & Interval \\
\hline$r_{i, j}$ & {$[1-3]$} & $q_{i}, i \in I_{1}$ & {$[50-100] \mathrm{kg}$} \\
$p_{i}, i \in I_{1}$ & {$[0.10-0.17] \mathrm{h}$} & $q_{i}, i \in I_{2}$ & {$[20-40] \mathrm{kg}$} \\
$p_{i}, i \in I_{2}$ & {$[0.05-0.10] \mathrm{h}$} & $q_{i}, i \in I_{3}$ & {$[3-6] \mathrm{kg}$} \\
\hline
\end{tabular}

TABLE 3: Comparison between CPLEX solver and PSO algorithm on small-scale instances.

\begin{tabular}{|c|c|c|c|c|c|c|}
\hline \multicolumn{2}{|c|}{ Instance } & \multicolumn{2}{|c|}{ CPLEX } & \multicolumn{3}{|c|}{ PSO } \\
\hline Group & ID & $F_{\text {CPLEX }}$ & $t_{\text {CPLEX }}$ & $F_{\mathrm{PSO}}$ & $t_{\mathrm{PSO}}$ & $\operatorname{Gap}_{1}(\%)$ \\
\hline \multirow{4}{*}{ ISG1 } & 1 & 1805.67 & 14.3 & 1874.69 & 10.7 & 3.82 \\
\hline & 2 & 1652.55 & 41.7 & 1663.95 & 10.4 & 0.69 \\
\hline & 3 & 1672.35 & 3.4 & 1729.20 & 10.5 & 3.40 \\
\hline & 4 & 1722.14 & 15.5 & 1788.40 & 11.9 & 3.85 \\
\hline \multirow{4}{*}{ ISG2 } & 1 & 2282.41 & 93.0 & 2446.26 & 20.7 & 7.18 \\
\hline & 2 & 2137.46 & 1232.6 & 2265.62 & 33.4 & 6.00 \\
\hline & 3 & 2265.72 & 1765.3 & 2360.77 & 35.1 & 4.20 \\
\hline & 4 & 2174.38 & 178.2 & 2305.10 & 33.2 & 6.01 \\
\hline \multirow{4}{*}{ ISG3 } & 1 & - & $>7200$ & 4059.08 & 61.2 & - \\
\hline & 2 & - & $>7200$ & 3423.35 & 61.1 & - \\
\hline & 3 & - & $>7200$ & 3691.92 & 61.8 & - \\
\hline & 4 & - & $>7200$ & 3502.65 & 59.2 & - \\
\hline Average & & & & & 34.1 & 4.39 \\
\hline
\end{tabular}

Notes: (1) $F_{\text {CPLEX }}$ represents the optimal solution obtained by CPLEX. $F_{\mathrm{PSO}}$ denotes the global best solution obtained by the PSO algorithm. (2) $t_{\mathrm{CPLEX}}$ and $t_{\mathrm{PSO}}$ are the computation times of CPLEX and PSO algorithm in seconds, respectively. (3) $\mathrm{Gap}_{1}=\left(F_{\mathrm{PSO}}-F_{\mathrm{CPLEX}}\right) / F_{\mathrm{CPLEX}}$.

TABle 4: Comparison between the rules and the PSO algorithm on large-scale instances.

\begin{tabular}{|c|c|c|c|c|c|}
\hline \multicolumn{2}{|c|}{ Instance } & \multicolumn{2}{|c|}{ Rules } & \multicolumn{2}{|c|}{ PSO } \\
\hline Group & ID & $F_{\text {Rules }}$ & $F_{\text {PSO }}$ & $t_{\mathrm{PSO}}$ & $\mathrm{Gap}_{2}(\%)$ \\
\hline \multirow{4}{*}{ ISG4 } & 1 & 8225.41 & 6572.28 & 151.6 & -25.15 \\
\hline & 2 & 7936.79 & 6211.87 & 153.6 & -27.77 \\
\hline & 3 & 7906.90 & 6754.96 & 159.2 & -17.05 \\
\hline & 4 & 7938.97 & 6103.34 & 151.9 & -30.08 \\
\hline \multirow{4}{*}{ ISG5 } & 1 & 11658.01 & 10372.02 & 350.6 & -12.40 \\
\hline & 2 & 12202.23 & 10307.83 & 383.2 & -18.38 \\
\hline & 3 & 12052.78 & 10003.89 & 360.2 & -20.48 \\
\hline & 4 & 12051.08 & 10206.68 & 375.5 & -18.07 \\
\hline \multirow{4}{*}{ ISG6 } & 1 & 15798.76 & 13160.92 & 675.3 & -20.04 \\
\hline & 2 & 15901.45 & 13622.51 & 636.4 & -16.73 \\
\hline & 3 & 15738.03 & 13781.96 & 673.1 & -14.19 \\
\hline & 4 & 15956.30 & 13181.95 & 629.1 & -21.05 \\
\hline Average & & & & 391.6 & -20.12 \\
\hline
\end{tabular}

Notes: (1) $F_{\text {Rules }}$ represents the feasible solution obtained by rules. $F_{\text {PSO }}$ denotes the global best solution obtained by the PSO algorithm. (2) $t_{\mathrm{PSO}}$ is the computation time of the PSO algorithm in seconds. (3) $\mathrm{Gap}_{2}=\left(F_{\mathrm{PSO}}-F_{\text {Rules }}\right) / F_{\text {Rules }}$. 


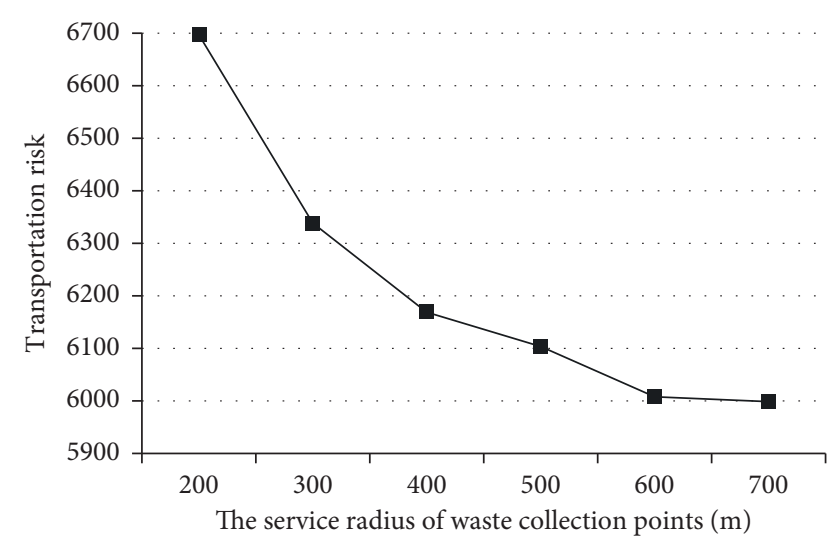

FIgURE 1: Sensitivity analysis about the service area of collection points.

transportation distance could be prolonged, and the transportation risk will be significantly increased, which may be beyond the scope of the assumption. In practice, the service area is usually limited within an appropriate range. The service area can be expanded into an appropriate range on the premise of safety.

Then we change the interval of the waste amount produced by facilities with collection points. In this analysis, the amount of waste produced by a facility with collection point is randomly selected from a specific range. The results are shown in Figure 2. Unsurprisingly, the total risk shows an upward trend with the increase of waste amount since fewer facilities could be assigned to a route.

The impact of the number of facilities with collection points in the network is also investigated by changing the number of facilities. The results are shown in Figure 3. More facilities in the network lead to higher potential transportation risk. We observe that the marginal risk decreases as the number of facilities with collection points increases. One possible reason is that more collection points may provide more choices for the collection of small facilities. Another reason for the marginal diminishing trend may relate to the increase in vehicle utilisation. When the number of routes is determined, more facilities with collection points will be assigned to a route. This can make better use of vehicles' capacity. However, when more facilities are included in the network, the vehicles may be overloaded, and thus, more routes are required in the network. There is no doubt that fewer facilities can lead to less risk; however, when the total number of routes is limited by budget or other reasons, sometimes the increasing need for waste collection can be satisfied with a slight increase in transportation risk by using the marginal diminishing effect.

6.3.2. Sensitivity Analysis about Collection Routes. The sensitivity analysis of collection routes focuses on the impact on shortening time windows and is conducted by changing the latest service time. Each task's earliest service time is 0 am. From Figure 4, we can see that when the latest service time changes from 10 am to $7 \mathrm{am}$, the total transportation

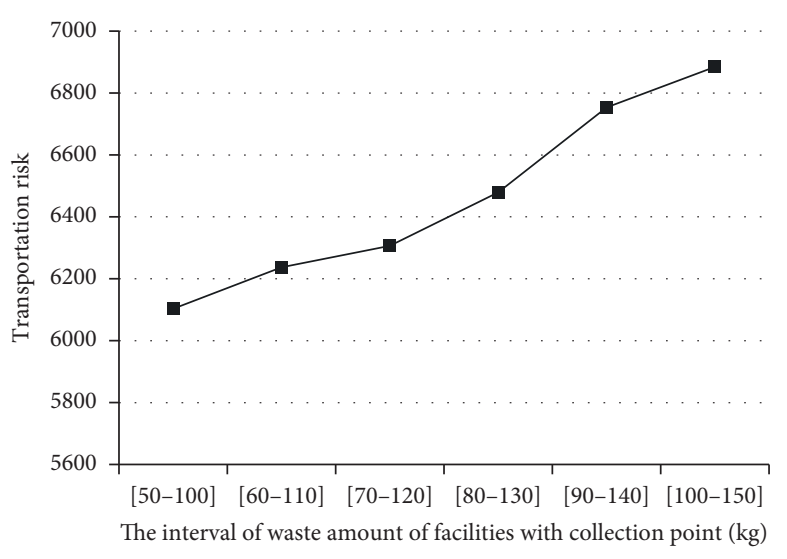

FIGURE 2: Sensitivity analysis about the amount of waste produced by facilities with collection points.

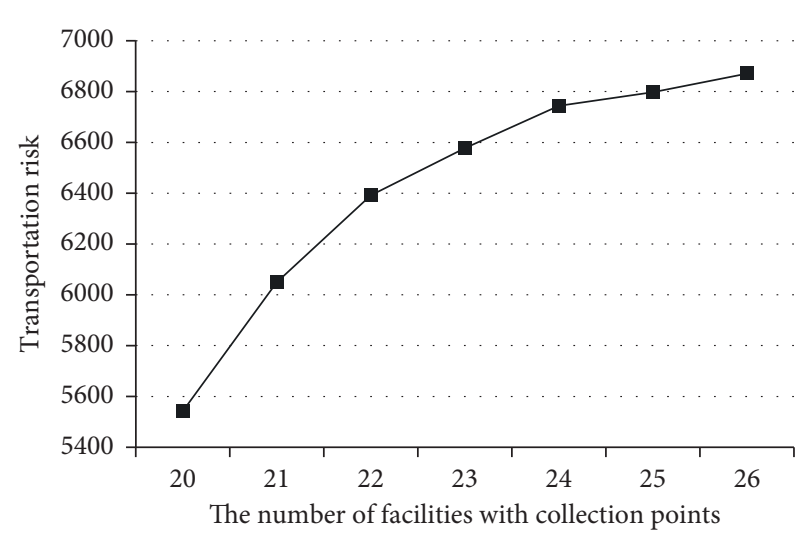

FIGURE 3: Sensitivity analysis about the number of facilities with collection points.

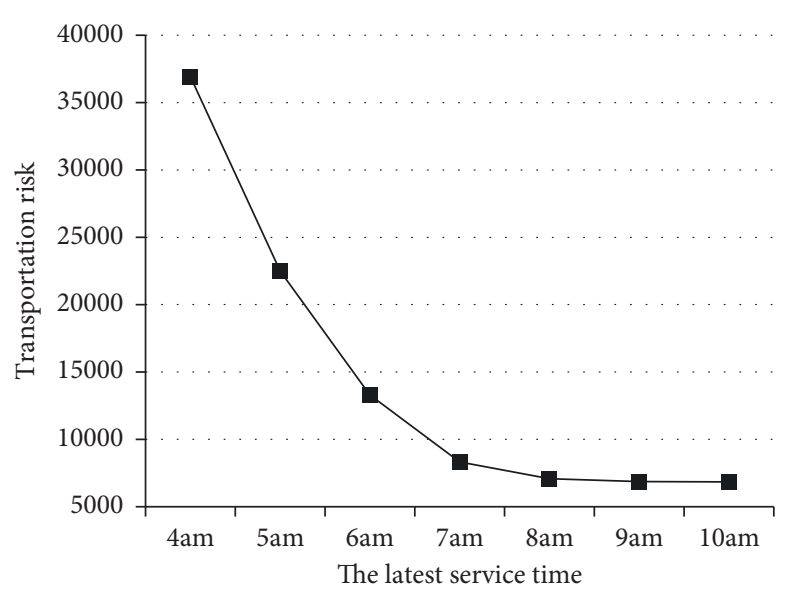

FIGURE 4: Sensitivity analysis about the tasks' latest service time.

risk is increased slightly. If the time windows are further shortened, the total transportation risk is sharply increased. This implies that there is slack in the time windows of ISG4, and operators can reduce the time windows with a slight increase in total risk. 


\section{Conclusion}

This paper studies the waste collection route optimisation in a healthcare system. The facilities in the healthcare system are classified into three categories according to the functions and scales, namely, facilities with collection points, facilities without collection points, and small facilities. A two-echelon collection mode is applied in the collection of small facilities where the waste of small facilities is transferred by the facilities with collection points. To formulate and solve the proposed problem, we developed a mixed-integer linear programming model with the objective of minimising the total transportation risk and designed a tailored PSO solution approach. The numerical experiments show the efficiency of the proposed algorithm, and sensitivity analysis is conducted to obtain some managerial implications from the perspective of collection points and routes.

The major contributions are summarised in the following aspects:

(1) This paper extends the waste collection research tothe healthcare system. A two-echelon collection mode is employed for the collection of small facilities, which provides a new method for the waste collection of the healthcare system.

(2) This paper develops a mixed-integer linear programming model and proposes a PSO solution approach with high computational efficiency. The proposed algorithm can provide a decision support tool for the proposed problem.

(3) The sensitivity analysis is conducted from the perspective of facilities with collection points and routes, and some insights are concluded.

However, the study contains some limitations. We only investigate the deterministic circumstance where the waste amount is known in advance and the transportation risk is predetermined. A stochastic optimisation where the amount of waste is randomly distributed may be more useful for the network operation. In addition, the online routing algorithm for dynamic road condition may work better in practice. To simplify the problem, we only abstract three types of facilities in the healthcare system. Future research can investigate some more complex system structures and other collection modes to broaden the research scope.

\section{Data Availability}

No data were used to support this study.

\section{Conflicts of Interest}

The authors declare that they have no conflicts of interest.

\section{Acknowledgments}

This research was supported by the National Key $R \& D$ Program of China (Grant no. 2018YFE0102700).

\section{References}

[1] Z. Wang, L. Huang, and C. X. He, "A multi-objective and multi-period optimization model for urban healthcare waste's reverse logistics network design," Journal of Combinatorial Optimization, vol. 7, 2019.

[2] M. Veenstra, K. J. Roodbergen, L. C. Coelho, and S. X. Zhu, “A simultaneous facility location and vehicle routing problem arising in health care logistics in The Netherlands," European Journal of Operational Research, vol. 268, no. 2, pp. 703-715, 2018.

[3] L. Delgado-Antequera, F. Pérez, A. G. Hernández-Díaz, and A. D. López-Sánchez, "An interactive biobjective method for solving a waste collection problem," Mathematical Problems in Engineering, vol. 2016, Article ID 5278716, 2016.

[4] V. R. Ghezavati and M. Beigi, "Solving a bi-objective mathematical model for location-routing problem with time windows in multi-echelon reverse logistics using metaheuristic procedure," Journal of Industrial Engineering International, vol. 12, no. 4, pp. 469-483, 2016.

[5] M. Zhang, S. Pratap, Z. Zhao, D. Prajapati, and G. Q. Huang, "Forward and reverse logistics vehicle routing problems with time horizons in B2C e-commerce logistics," International Journal of Production Research, vol. 34, pp. 1-20, 2020.

[6] P. Sitek, J. Wikarek, K. Rutczyńska-Wdowiak, G. Bocewicz, and Z. Banaszak, "Optimization of capacitated vehicle routing problem with alternative delivery, pick-up and time windows: a modified hybrid approach," Neurocomputing, vol. 423, pp. 670-678, 2021.

[7] J. Van Engeland and J. Beliën, "Tactical waste collection: column generation and mixed integer programming based heuristics," OR Spectrum, vol. 28, 2020.

[8] M. A. Hannan, M. Akhtar, R. A. Begum, H. Basri, A. Hussain, and E. Scavino, "Capacitated vehicle-routing problem model for scheduled solid waste collection and route optimization using PSO algorithm," Waste Management, vol. 71, pp. 31-41, 2018.

[9] L. Delgado-Antequera, M. Laguna, J. Pacheco, and R. Caballero, "A bi-objective solution approach to a realworld waste collection problem," Journal of the Operational Research Society, vol. 71, no. 2, pp. 183-194, 2020.

[10] R. Montagné, M. Gamache, and M. Gendreau, "A shortest path-based algorithm for the inventory routing problem of waste vegetable oil collection," Journal of the Operational Research Society, vol. 70, no. 6, pp. 986-997, 2019.

[11] M. Taslimi, R. Batta, and C. Kwon, "Medical waste collection considering transportation and storage risk," Computers \& Operations Research, vol. 120, Article ID 104966, 2020.

[12] E. B. Tirkolaee, P. Abbasian, and G.-W. Weber, "Sustainable fuzzy multi-trip location-routing problem for medical waste management during the COVID-19 outbreak," Science of The Total Environment, vol. 756, Article ID 143607, 2021.

[13] H. Li, J. Yuan, T. Lv, and X. Chang, "The two-echelon timeconstrained vehicle routing problem in linehaul-delivery systems considering carbon dioxide emissions," Transportation Research Part D: Transport and Environment, vol. 49, pp. 231-245, 2016.

[14] J. Zhang, Y. Zhao, W. Xue, and J. Li, "Vehicle routing problem with fuel consumption and carbon emission," International Journal of Production Economics, vol. 170, pp. 234-242, 2015.

[15] J. C. Molina, I. Eguia, and J. Racero, "Reducing pollutant emissions in a waste collection vehicle routing problem using a variable neighborhood tabu search algorithm: a case study," The New England Journal of Medicine, vol. 27, no. 2, pp. 253-287, 2019. 
[16] H. Farrokhi-Asl, A. Makui, A. Jabbarzadeh, and F. Barzinpour, "Solving a multi-objective sustainable waste collection problem considering a new collection network," Operational Research, vol. 20, no. 4, pp. 1977-2015, 2020.

[17] Q. Wei, Z. Guo, H. C. Lau, and Z. He, “An artificial bee colony-based hybrid approach for waste collection problem with midway disposal pattern," Applied Soft Computing, vol. 76, pp. 629-637, 2019.

[18] H. L. Wu, F. M. Tao, and B. Yang, "Optimization of vehicle routing for waste collection and transportation," International Journal of Environmental Research and Public Health, vol. 17, p. 26, 2020.

[19] R. Aringhieri, M. bruglieri, F. malucelli, and M. Nonato, "A special vehicle routing problem arising in the optimization of waste disposal: a real case," Transportation Science, vol. 52, pp. 277-299, 2017.

[20] H. Jammeli, M. Argoubi, and H. Masri, "A Bi-objective stochastic programming model for the household waste collection and transportation problem: case of the city of Sousse," Operational Research, vol. 162, no. 3, 2019.

[21] K. Mekamcha, M. Souier, H. N. Bessenouci, and M. Bennekrouf, "Two metaheuristics approaches for solving the traveling salesman problem: an algerian waste collection case," Operational Research, vol. 4, 2019.

[22] P. De Bruecker, J. Beliën, L. De Boeck, S. De Jaeger, and E. Demeulemeester, "A model enhancement approach for optimizing the integrated shift scheduling and vehicle routing problem in waste collection," European Journal of Operational Research, vol. 266, no. 1, pp. 278-290, 2018.

[23] S. Kargar, M. Pourmehdi, and M. M. Paydar, "Reverse logistics network design for medical waste management in the epidemic outbreak of the novel coronavirus (COVID-19)," Science of The Total Environment, vol. 746, Article ID 141183, 2020.

[24] K. Kang, X. Wang, and Y. Ma, "A collection-distribution center location and allocation optimization model in closedloop supply chain for Chinese beer industry," Mathematical Problems in Engineering, vol. 2017, Article ID 7863202, 2017.

[25] X. F. Ji, J. S. Pan, S. C. Chu, P. Hu, Q. W. Chai, and P. Zhang, "Adaptive cat swarm optimization algorithm and its applications in vehicle routing problems," Mathematical Problems in Engineering, vol. 2020, Article ID 1291526, 14 pages, 2020.

[26] L. Zhen, "Modeling of yard congestion and optimization of yard template in container ports," Transportation Research Part B: Methodological, vol. 90, pp. 83-104, 2016.

[27] N. Umang, M. Bierlaire, and I. Vacca, "Exact and heuristic methods to solve the berth allocation problem in bulk ports," Transportation Research Part E: Logistics and Transportation Review, vol. 54, pp. 14-31, 2013.

[28] L. Zhen, "Tactical berth allocation under uncertainty," European Journal of Operational Research, vol. 247, no. 3, pp. 928-944, 2015.

[29] L. Zhen, E. P. Chew, and L. H. Lee, “An integrated model for berth template and yard template planning in transshipment hubs," Transportation Science, vol. 45, no. 4, pp. 483-504, 2011.

[30] L. Zhen, Z. Liang, D. Zhuge, L. H. Lee, and E. P. Chew, "Daily berth planning in a tidal port with channel flow control," Transportation Research Part B: Methodological, vol. 106, pp. 193-217, 2017.

[31] B. Yao, B. Yu, P. Hu, J. Gao, and M. Zhang, "An improved particle swarm optimization for carton heterogeneous vehicle routing problem with a collection depot," Annals of Operations Research, vol. 242, no. 2, pp. 303-320, 2016.
[32] L. Zhen, Z. Xu, K. Wang, and Y. Ding, "Multi-period yard template planning in container terminals," Transportation Research Part B: Methodological, vol. 93, pp. 700-719, 2016.

[33] I. H. Dridi, E. Ben Alaia, P. Borne, and H. Bouchriha, "Optimisation of the multi-depots pick-up and delivery problems with time windows and multi-vehicles using PSO algorithm," International Journal of Production Research, vol. 58, pp. 4201-4214, 2020.

[34] J. Kennedy and R. Eberhart, "Particle swarm optimization," in Proceedings of the ICNN'95 - International Conference on Neural Networks, Perth, Australia, December 1995. 